\title{
Childhood Central Nervous System Neuroblastoma
}

National Cancer Institute

\section{Source}

National Cancer Institute. Childhood Central Nervous System Neuroblastoma. NCI

Thesaurus. Code C114775.

A neuroblastoma that arises from the cerebral hemispheres and occurs during childhood. 